\title{
BUSINESS AGILITY: DESAFIOS EM UM PROJETO DE UM APLICATIVO MÓVEL PARA O SETOR DE SERVIÇOS FINANCEIROS DIGITAIS
}

Breno Mantovani (breno.mantovani@outlook.com)

Camila de Araujo (amiladearaujo@ufu.br) - FAGEN, Universidade Federal de Uberlândia (UFU).

\section{RESUMO}

O cenário atual de inovações e mudanças tecnológicas está caracterizado pela transformação digital. O setor de serviços financeiros foi um dos que conduziu sua estratégia em direção ao digital, por perceber que seus clientes migraram suas atividades tradicionais para ambientes digitais. Como estratégia para apoiar os desafios do momento, o Business Agility surge como uma possibilidade de intensificar a efetividade na obtenção dos resultados por meio da agilidade alinhada às novas tecnologias. Assim, este trabalho analisa os fatores do Business Agility em um estudo de caso sobre um projeto de desenvolvimento de uma aplicação digital móvel no setor de serviços financeiros digitais, bem como os desafios e oportunidades encontrados. Concluiu-se que a aplicação de conceitos do Business Agility pôde auxiliar no sucesso do projeto, que se encontra no cenário de transformação digital e de desenvolvimento de novas estratégias de competitividade, a partir da geração de uma conexão estratégica entre os diferentes níveis hierárquicos, com foco no processo e entrega de valor para o cliente.

Palavras chave: agilidade; transformação digital; pagamentos eletrônicos; estratégia; aplicações

digitais 


\section{INTRODUÇÃO}

Organizações em quase todos os setores tem dado ênfase à transformação digital, em busca de responder às demandas do mercado e maximizar seus resultados (SIACHOU; VRONTIS; TRICHINA, 2021). Souza et al. (2013) discorrem sobre o crescente uso da tecnologia móvel e como tal fato concebe um novo perfil de entrega de valor ao usuário e interação com esse ao citar o grande número de pessoas que utilizam a mobilidade devido ao acesso às informações de maneira rápida e personalizada. Dados estatísticos de uma das mais renomadas empresas de análises de aplicativos, a App Annie (2020), indicam que no período de 2016 a 2019 o número de downloads de aplicações digitais cresceu em 45\%, com um total de 204 bilhões de downloads em 2019, e o mercado de serviços financeiros, um dos mais expressivos, apresentou mais de 1 trilhão de acessos no ano 2019 em atividades que vão do gerenciamento de ações e acesso à bancos digitais ao uso de aplicativos de pagamento, denotando parcela importante no desenvolvimento de aplicações digitais.

A difusão da tecnologia aos usuários repercute então no impacto direto no mundo dos negócios, onde empresas passam a buscar maneiras de traduzir demandas em produtos digitais aos seus clientes. A maneira como essas empresas se transformam e aprendem a responder às mudanças de estratégia rapidamente as prepara para prosperar em períodos de volatilidade e inovação e a garantir demandas contínuas (DOZ; KOSONEN, 2010).

Nesse contexto, a agilidade organizacional, mais conhecida pelo termo em inglês Business Agility, se propõe como uma abordagem para intensificar a efetividade da organização com base na inovação dos processos operacionais internos, envolvendo os clientes em suas atividades, enquanto potencializa as capacidades dos parceiros na rede de negócios (VAN OOSTERHOUT et al., 2006). Entender quais são os desafios no desenvolvimento de seus produtos e garantir que o planejamento analítico esteja estruturado em todos os níveis organizacionais pode gerar crescimento e estabilidade nos mais diferentes cenários econômicos.

Partindo do exposto, a aplicação dos conceitos de Business Agility no desenvolvimento de produtos digitais do segmento financeiro, que representa um mercado considerável onde os consumidores estão migrando cada vez mais suas atividades tradicionais para ambientes digitais, pode ser importante para intensificar a eficiência na busca por melhores resultados. Assim, o presente estudo pretende analisar os conceitos de Business Agility em um projeto de desenvolvimento de uma aplicação digital móvel, tendo como base um estudo de caso no setor de serviços financeiros digitais, buscando apresentar e analisar dados e fatores chave que 
contribuem ou não para o sucesso dessa abordagem e, consequentemente os desafios e contribuições para o projeto do aplicativo móvel estudado.

\section{REVISÃO TEÓRICA}

\subsection{O Setor de Produtos e Serviços Digitais e a Estratégia Competitiva}

Um estudo realizado pela GSMA (2020) indicou que o mercado de produtos e serviços digitais voltado para aplicativos móveis representou 4,7\% do PIB em todo o mundo, totalizando US\$ 4.1 trilhões de dólares em valor econômico agregado, além de contribuir substancialmente com empregos diretos e indiretos em todo o globo, arrecadando US\$ 490 bilhões de dólares ao setor público. Em 2019, empresas voltadas para o desenvolvimento digital apresentaram um valor de mercado de 544 bilhões de dólares, 6.5 vezes maior que empresas que não possuíram o mesmo objetivo (APP ANNIE, 2020). É esperado que até 2024 a parcela de participação do mercado de produtos e serviços digitais móveis represente $4,9 \%$ do PIB para a economia global, totalizando um valor aproximado de US\$ 5 trilhões de dólares visto que que cada vez mais diversos países em todo o mundo se beneficiam das melhorias de pesquisa e eficiência provocadas pelo aumento da aceitação de serviços móveis (GSMA, 2020). O acesso às informações de maneira rápida e personalizada é fator chave na concepção do novo perfil de entrega de valor e interação ao consumidor, e o crescente uso da tecnologia móvel contribui fortemente na criação deste perfil consumidor, dado o número expressivo de pessoas que utilizam a mobilidade móvel em todo o mundo devido a sua eficiência e praticidade (SOUZA et al., 2013).

Usai et al. (2021) citam vários acadêmicos que reconhecem que as tecnologias digitais podem fazer a diferença na obtenção de vantagem competitiva para a iniciativa estratégica e para a agilidade da empresa, baseada na consistência entre as tecnologias digitais e as capacidades dinâmicas da empresa. Percebe-se então que o grande diferencial das novas empresas e também de empresas tradicionais no setor de produtos e serviços está na capacidade de atender às novas demandas, onde a presença digital se destaca como característica fundamental de competitividade na definição de atratividade ao consumidor e potencial de rentabilidade aos investidores (GFT GROUP, 2019). 


\subsection{Business Agility}

A agilidade organizacional, mais conhecida pelo seu termo em inglês Business Agility, é abordada tanto no âmbito acadêmico quanto empresarial/comercial. Academicamente, é descrita por van Oosterhout et al. (2006) como a capacidade da organização de sentir incertezas e mudanças externas e internas e de responder a elas, com base na inovação dos processos operacionais internos, envolvendo o cliente em atividades de exploração e explotação, enquanto potencializa as capacidades de parceiros na rede de negócios. A definição dada pela Scaled Agile Inc. (2020), organização voltada para o mercado comercial, é a capacidade de competir respondendo rapidamente às mudanças e oportunidades do mercado com soluções inovadoras utilizando as práticas Lean e Agile para oferecer produtos e serviços mais rápido que a concorrência. Nafei (2016) complementa que o conceito considera a capacidade de sobreviver e prosperar em um ambiente competitivo de mudança contínua e imprevisível ao reagir de maneira rápida e eficaz em mercados de constante mudança com uma estratégia de gerenciamento proativo que mantenha os recursos da organização e atenda aos desejos dos clientes em tempo hábil impulsionado por produtos e serviços bem definidos. Rigby, Elk e Berez (2020) explicam que não basta ter equipes ágeis para transformar as experiências dos clientes e superar os concorrentes continuamente, é preciso que uma empresa adote esses princípios em todos os níveis organizacionais, inclusive nos de alto executivos.

Construir uma empresa ágil significa encontrar o equilíbrio entre padronizar operações e buscar inovações de modo que a estratégia não se torne demasiadamente estática e oculta ou extremamente arriscada e caótica para seus clientes e colaboradores (RIGBY; ELK; BEREZ, 2020). Zacarias, Martins e Gonçalves (2017) identificam no âmbito organizacional da agilidade operacional a atuação de níveis de processo no qual a gerência pode adicionar ou redesenhar processos ou produtos para aproveitar ou explorar as condições do ambiente dinâmico. O conceito proposto por Zacarias, Martins e Gonçalves (2017) é composto por quatro componentes que possibilitam uma visão detalhada do processo, sendo eles: 1Reconfigurabilidade, como capacidade de se adaptar às mudanças, adicionar ou alterar processos de negócios novos ou aprimorados; 2- Capacidade de resposta, relacionada ao fator tempo e a capacidade de reagir às mudanças de maneira oportuna; 3- Adaptabilidade dos colaboradores, ao reconhecer que a agilidade requer pessoas com conhecimento e capacidade para responder as condições dinâmicas, e, 4 - Visão centrada no processo, essa direcionada aos gerentes e a capacidade de entender o fluxo operacional de ponta a ponta para a tomada de 
decisões estratégicas, uma vez que os processos de negócio ultrapassam departamentos funcionais.

Assim, o sucesso estratégico definido atinge seu objetivo apenas se dispuser de coerência organizacional, em que existe uma conexão estratégica feita em A, com a coordenação no método B e a operação feita do método C. Sem isso lacunas começam a aparecer na organização e se difundem como ineficiência nos processos e produtos.

\section{METODOLOGIA}

Esta pesquisa é de natureza aplicada, de caráter exploratório e foi desenvolvida com base em uma abordagem qualitativa a partir do procedimento de estudo de caso único. Tal procedimento foi escolhido por ser adequado para avaliar o acontecimento de um fenômeno dentro do seu contexto, sendo assim uma pesquisa de cunho empírico (YIN, 2014). O estudo foi realizado em uma companhia multinacional que desenvolve produtos para pagamentos digitais, tendo como unidade de análise o projeto de desenvolvimento de uma aplicação digital móvel. O projeto ocorreu dentro do prazo de um ano, entre 2019 e 2020. Como instrumento de coleta de dados, foi utilizado um roteiro de entrevista com perguntas semiabertas, observações e análise dos artefatos do projeto. Os participantes da equipe do projeto da unidade brasileira foram entrevistados.

Como base teórica, realizou-se também uma revisão bibliográfica não exaustiva de conceitos relacionados ao Business Agility e estratégia organizacional na concepção de aplicações móveis digitais sendo que as buscas de materiais foram realizadas em bases de dados acadêmico científicas, sites e instituições de classe, além da análise de materiais impressos e eletrônicos.

\section{RESULTADOS}

A empresa onde ocorreu o projeto estudado por este trabalho oferece serviços de pagamentos eletrônicos desde sua criação por meio de soluções financeiras a todos os tipos e tamanhos de negócio. Sua estrutura é composta por uma matriz situada na Alemanha e diversas subsidiarias em todo o mundo. $\mathrm{O}$ conselho administrativo se concentra na matriz, cabendo a esse definir a direção estratégica da organização, repassar objetivos aos executivos seniores de cada divisão e informar o plano às subsidiárias e seus respectivos executivos globais. A cada unidade subsidiária cabe definir sua estratégia de negócio em concordância com os cenários locais de competitividade e mercado e garantir que as definições estejam alinhadas ao plano 
previamente definido pelo conselho administrativo. A partir da estratégia definida, os executivos estipulam os objetivos a serem alcançados por cada divisão, tais objetivos são passados aos heads, líderes de departamento que estipulam o escopo e a finalidade a ser alcançada pela liderança de cada equipe. Essa última delibera os objetivos necessários a serem obtidos por cada membro de seu time. Por se tratar de uma grande fintech, a empresa possui uma cartela de clientes com tamanhos de negócio variados e os classifica em grandes ou pequeno/médio, citados neste estudo pelos termos big merchant e SME merchant.

Os serviços de gerenciamento e monitoramento das atividades financeiras são oferecidos aos clientes em duas versões de plataformas e dependem da classificação atribuída previamente ao merchant. A classificação estipulada é que direciona o merchant para uma plataforma ou outra independentemente de ambas pertencerem à mesma ferramenta. Na visão estratégica da empresa, a versão clássica é desenvolvida para grandes clientes, permitindo a esses se beneficiarem de toda a prateleira de serviços e produtos oferecidos para o comércio eletrônico, enquanto a versão light é oferecida aos SME merchants, para que esses obtenham a facilidade em acompanhar suas transações em tempo real por meio de uma interface mais objetiva e personalizada ao seu negócio. Por possuir cunho global, diversos projetos existentes podem atender a um mesmo mercado ou até mesmo objetivar um mesmo resultado em diferentes regiões do mundo. Desta maneira, para que o trabalho aconteça de modo fluído, a empresa trabalha com times e projetos locais e times e projetos globais. Essa característica é relativa ao modo pelo qual uma área se relaciona com a outra, bem como os recursos entre times, concebendo por assim níveis de autoridade e relacionamento cruzados entre equipes e projetos. O projeto analisado foi desenvolvido na unidade do Brasil por um time global, com o objetivo de desenvolver uma aplicação móvel para usuários do mercado Europeu.

\subsection{O Desenvolvimento da Aplicação Digital para o Portal Empresarial}

O contexto do projeto estudado foi iniciado como parte da estratégia de negócios para manter a competitividade, onde os executivos da divisão de gerenciamento de clientes (gerência sênior) definiram a necessidade de desenvolvimento de um aplicativo para o maior portal da companhia, que permitisse para clientes acesso a transações e dados de suas contas por um dispositivo móvel. O propósito consistia em manter a competitividade ao atender todos os tipos de usuários, big e SME merchants. Paralelamente ao serviço, havia também o objetivo de coletar dados para que a empresa pudesse utilizar internamente para a tomada de decisões 
em novas estratégias de negócio e produtos. Assim, esse era um projeto interno, com a necessidade determinada por uma decisão vertical, da gerência para a operação.

Foram observados os seguintes envolvidos diretamente no projeto:

- Gerência sênior: responsável pela estratégia gerencial do negócio com o produto proposto, localizada fora do Brasil. Concentra-se em um nível estratégico de negócio;

- Escritório de projetos (Project Management Office - PMO): responsável pelo contato direto com a gerência de clientes, de forma a alinhar expectativas e necessidades.

- Equipe do projeto: formada por gerente de projeto (Project Manager - PM) e time de desenvolvimento, composto por dois desenvolvedores, sendo um para a plataforma Android e outro para a iOS, e um designer de experiência do usuário. Após o primeiro mês de projeto, um analista de negócios foi incluído, para traduzir as necessidades de negócio em requisitos técnicos ao produto. Toda a equipe concentra-se em um nível operacional.

A partir de discussões entre a gerência sênior e a equipe do projeto, bem como reuniões entre a equipe e PMO, o objetivo definido foi oferecer a solução ao público SME merchant, mas com o objetivo final de escalar a aplicação para todos os tamanhos de negócio da empresa. Assim, o esperado era que ocorresse a fase de concepção, com o detalhamento de soluções técnicas e de design, na qual é feito o alinhamento de como a construção deve acontecer, com cenários e detalhes sobre possibilidades e limitações no decorrer do desenvolvimento. Em seguida, inicia-se a fase de desenvolvimento, onde é gerado a versão do produto chamada de MVP (Minimum Viable Product). Por fim, a fase do ambiente de produção seria iniciada.

Foi observado que o PM era responsável por prover detalhes ao escritório de projetos, enquanto o time de desenvolvimento se concentrou na criação, testes e usabilidade do produto a ser desenvolvido. A liderança do PMO foi responsável por alinhar as expectativas e entregas com a gerência sênior e por consultar o PM quando necessário.

No entanto, mesmo após os trâmites iniciais, reunião de kick-off e fase de concepção com as soluções técnicas e de design, o time de desenvolvimento apresentou vários questionamentos relacionados a possiblidade de expansão do público do produto, tais como se o time deveria concentrar seus esforços nos SME ou todos os negócios; se o time poderia adicionar comerciantes da versão clássica nas etapas iniciais de desenvolvimento; se a equipe poderia reutilizar o design e os recursos da versão SME para a clássica.

Assim, ficou claro que as expectativas da gerência sênior estavam desconectadas do cenário operacional técnico dado que, embora o objetivo final proposto de expandir para todos os tamanhos de negócio fosse na visão gerencial uma atividade atrativa, haviam diferentes 
configurações técnicas, que resultavam em múltiplas maneiras de desenvolver a solução inicialmente, ou seja, a escalabilidade que a gerencia sênior entendia como algo fácil de ser realizado, em termos técnico não era algo tão simples. Ainda assim, a gerência sênior solicitou ao time continuar o desenvolvimento dos recursos mandatórios da aplicação e relevar a falta de clareza das circunstâncias iniciais.

Após 7 meses, devido ao desalinhamento das visões, o MVP não estava pronto e membros do time acabaram por solicitar desligamento da equipe e da organização, justificando o baixo senso de pertencimento e falta de clareza do objetivo final de suas tarefas.

A empresa então providenciou uma visita local da gerência sênior para alinhamento, quando ficou decidida uma alteração do objetivo explícito: trazer todos os tipos de merchants à aplicação, adaptando os recursos previamente desenvolvidos para garantir uma versão mínima viável da aplicação na loja de aplicativos móvel.

Durante a segunda fase de desenvolvimento que sucedeu o diálogo direcionado aos pontos de dor da equipe, a gerência sênior tratou de tomar ações para revisar todo o trabalho desenvolvido, reconhecer falhas em comunicação entre os times e diferentes níveis hierárquicos, corrigir erros e buscou focar seus esforços em reconstruir o time de operações. Em poucos meses o projeto retomou o andamento e a estratégia foi tratada de maneira mais clara e colaborativa, na qual a gerência buscou compartilhar sua visão de negócio e permitiu que a operação fomentasse feedbacks para iniciar uma discussão, se necessário, em cenários nos quais a direção almejada não fosse tecnicamente viável como proposto. Com a ação da gerência de redefinir um novo plano de entrega, com novas datas e um escopo mais fechado, possibilitou que a equipe de desenvolvimento reduzisse drasticamente os cenários possíveis na aplicação. Com tal alinhamento a segunda etapa avançou para a entrega do produto nos ambientes de produção em 3 meses e a gerência concretizou a estratégia proposta no início do projeto.

Foi possível observar que o gestor, como líder estrategista, necessita encarar a estratégia como fator coletivo e compreensível, bem como alinhada essa à visão de cultura, pois isso proporcionará um ambiente colaborativo e aberto a erros e acertos permitindo que as pessoas traduzam seus esforços e cocriem um produto ou serviço.

A mudança para uma postura aberta possibilitou que ajustes fossem realizados, ouvindo tanto as expectativas da gerência no papel de cliente como da equipe, mantendo o cliente como ponto central no processo de tomada de decisão. O ambiente mais colaborativo auxiliou na resolução de dúvidas e abriu espaço para o diálogo entre diferentes níveis hierárquicos. 
Os recursos, a partir do entendimento do real motivo de seus esforços, conseguiram pensar de maneira inovadora e voltada para resultados.

\subsection{Análise na perspectiva ao Business Agility}

Considerando os fatores apresentados por Zacarias, Martins e Gonçalves (2017), no caso apresentado foi possível perceber que, embora a gerência tenha concebido o projeto de desenvolvimento da aplicação móvel como uma estratégia eficiente para alcançar os resultados esperados pela companhia, não havia engajamento e concordância entre todos os níveis envolvidos, ou seja, uma falha no fator "visão centrada no processo", principalmente da gerência para a operação. Foi possível observar também a necessidade do fator "Adaptabilidade dos colaboradores", quando da necessidade de inclusão do analista de negócios no time de desenvolvimento, após um mês do início do projeto. É dever da gerência, ao se trabalhar com os conceitos de Business Agility, ter uma visão centrada no processo e com capacidade para entender o fluxo operacional de ponta a ponta para a tomada de decisões, bem como entender quem são as pessoas do time e como cada posição contribui para a entrega dos resultados finais esperados, e, em cenários de déficit despertar os questionamentos percebidos acerca da falta de um conhecimento ou posição especifica na equipe.

Sobre o fator "Capacidade de resposta", foi possível observar que, mesmo após sete meses de andamento do projeto, o time não conhecia seu usuário final, o que gerou atrasos no desenvolvimento do projeto. O Business Agility como conceito elucida o papel da gerência em atuar nessas situações, adicionando ou redesenhando processos ou produtos se o objetivo for alcançar o entendimento de pontos de dor e necessidades em tempo hábil. A visita da gerência sênior na unidade do Brasil foi fator chave para que a necessidade de mudança fosse percebida. O exercício de colocar todos os níveis hierárquicos em uma única sessão elaborada de maneira transparente e respeitosa, mas direcionada aos maiores pontos de dor frente ao cenário de baixa entrega de resultados evitou que a informação persistisse a ser passada e recebida de maneira a salvaguardar interesses ou ocultar falhas de decisões previamente tomadas. A disponibilidade e atenção da gerência para fomentar novas discussões sobre o alcance dos próximos passos garantiu que os membros da equipe trabalhassem de maneira objetiva e comprometida e ainda garantiu que o time se sentisse ouvido e indispensável para o resultado final esperado. Tal prática buscou o equilíbrio entre padronizar operações e buscar inovações, de modo que a estratégia não se tornasse desconhecida entre colaboradores e 
clientes. Observou-se assim os fatores "Capacidade de reposta" e "Reconfigurabilidade" como importantes para o sucesso do projeto.

Portanto, os conceitos e fatores associados ao Business Agility auxiliaram na concepção de um produto aderente às necessidades de mercado, alinhado a estratégia final esperada de maneira eficiente e ativa ao negócio, conforme o conceito apresentado por Rigby, Elk e Berez (2020).

\section{CONCLUSÃO}

Este trabalho buscou analisar os conceitos de Business Agility em um projeto de desenvolvimento de uma aplicação digital móvel. Observou-se que, sem cultura alinhada internamente houve falha na relação de pertencimento e a falta de tal postura impedia que o negócio se adaptasse à novos métodos, sem romper antigos padrões e impossibilitando que a transformação ágil suceda resultados rápidos inovadores perante os concorrentes.

Concluiu-se que os conceitos de Business Agility puderam auxiliar no sucesso do projeto do novo produto, no cenário de transformação digital e de desenvolvimento de novas estratégias de competitividade, oferecendo uma conexão estratégica entre os diferentes níveis hierárquicos, com foco no processo e entrega de valor para o cliente.

As limitações deste estudo referem-se ao estudo de um único caso, bem como a característica do caso estudado de apresentar um cliente interno e que, ao mesmo tempo, atuou no nível gerencial. Assim, considera-se como possibilidade para estudos futuros, a realização de estudos de diferentes cenários para avaliar as dificuldades e desafios de adaptação das organizações em seus projetos de novos produtos.

\section{REFERÊNCIAS}

APP ANNIE (Org.). State of Mobile 2020. 2020. Disponível em: < https://www.appannie.com/en/go/state-of-

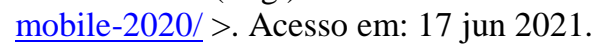

DOZ, Y.; KOSONEN, M. Fast! Como Usar a Agilidade Estratégica para Vencer. Bookman: Porto Alegre. 2010.

GFT GROUP. 11 GFT User Group - Business Agility. 2019. Elaborado Por Silvia Ura. Disponível em: < https://blog.gft.com/br/2019/11/07/11o-gft-user-group-business-agility/ >. Acesso em: 17 jun 2021.

GSMA. The Mobile Economy 2020. Londres: Gsma Intelligence, 2020. 57 p. Disponível em: < https://www.gsma.com/mobileeconomy/wp-content/uploads/2020/03/GSMA_MobileEconomy2020_Global.pdf >. Acesso em: 10 junho 2021.

NAFEI, Wageeh. Organizational Agility: the key to organizational success.: The Key to Organizational Success. International Journal of Business and Management, v. 5, n. 11, p. 296-309, 2016.

RIGBY, D. K.; ELK, S.; BEREZ, S. The Agile C-Suite. Harvard Business Review, 2020. 
SCALED AGILE INC. Business Agility. 2020. Disponível em: < https://www.scaledagileframework.com/business-agility/ >. Acesso em: 20 maio 2021.

SIACHOU, E.; VRONTIS, D.; TRICHINA, E. Can traditional organizations be digitally transformed by themselves? The moderating role of absorptive capacity and strategic interdependence. Journal of Business Research, v. 124, p. 408-421, 1 jan. 2021.

SOUZA, R. C. et al. Processo de criação de um aplicativo móvel na área de odontologia para pacientes com necessidades especiais. Rev. ABENO [online]. vol.13, n.2, p. 58-61, 2013.

USAI, A. et al. Unveiling the impact of the adoption of digital technologies on firms' innovation performance. Journal of Business Research, v. 133, p. 327-336, 2021.

VAN OOSTERHOUT, M., WAARTS, E., VAN HECK, E., VAN HILLEGERSBERG, J. Business agility: need, readiness and alignment with IT-strategies. In K. C. Desouza (Ed.), Agile Information Systems: Conceptualization, Construction and Management (pp. 52-69). Butterworth Heinemann, 2006.

YIN, R. K. Estudo de caso: planejamento e métodos. 5a ed. Porto Alegre: Bookman, 2014.

ZACARIAS, M.; MARTINS, P. V.; GONÇAlVES, A. An Agile Business Process and Practice Meta-model, Procedia Computer Science, v 121, p. 170-177, 2017. 